\title{
Advances on research epigenetic change of hybrid and polyploidy in plants
}

\author{
Zhiming Zhang ${ }^{\dagger}$, Jian $\mathrm{Gao}^{\dagger}$, Luo Mao, Qin Cheng, Zeng xing Li Liu, Haijian Lin, Yaou Shen, \\ Maojun Zhao and Guangtang Pan*
}

Maize Research Institute, Sichuan Agricultural University, Xinkang road 46, Ya’an, Sichuan 625014, People’s Republic of China.

Accepted 15 April, 2011

\begin{abstract}
Hybridization between different species, and subsequently polyploidy, play an important role in plant genome evolution, as well as it is a widely used approach for crop improvement. Recent studies of the last several years have demonstrated that, hybridization and subsequent genome doubling (polyploidy) often induce an array of variations that could not be explained by the conventional genetic paradigms. A large proportion of these variations are epigenetic in nature. Epigenetic can be defined as a change of the study in the regulation of gene activity and expression that are not driven by gene sequence information. However, the ramifications of epigenetic in plant biology are immense, yet unappreciated. In contrast to the ease with which the DNA sequence can be studied, studying the complex patterns inherent in epigenetic poses many problems. In this view, advances on researching epigenetic change of hybrid and polyploidy in plants will be initially set out by summarizing the latest researches and the basic studies on epigenetic variations generated by hybridization. Moreover, polyploidy may shed light on the mechanisms generating these variations. These advances will enhance our understanding on their evolutionary significances, as well as enable us to utilize these variations more effectively than before in crop breeding.
\end{abstract}

Key words: Epigenetic change, hybridization and polyploidy, evolution, crop breeding.

\section{INTRODUCTION}

In plants, hybrid speciation is frequently associated with polyploidy (allopolyploids) and the cases where new hybrid species share the same ploidy as their parents (homoploidy hybrid speciation) has also been described. Hybridization between different species and subsequently, polyploidy play an important role in plant genome evolution, as well as it is a widely used approach for crop improvement (Grant-Downton and Dickinson, 2006).

Recent studies have revealed that, there also exists a different type of variation that is not based on DNA sequence, named epigenetic variation. Accordingly, hybridization or polyploidy is found to be often associated with epigenetic variations. These epigenetic variations often transgress the laws of classical heredity, in that it is Lamarck's inheritance of acquired characters. It clearly

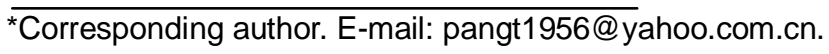

†These authors contributed equally to this work. suggests that, the non-additive change in genome size that occurred during allopolyploidization may represent a preprogrammed adaptive response to genomic stress caused by hybridization and allopolyploidy, which serves to stabilize polyploidy genomes (Ozkan et al., 2003). Recent transcriptomic studies of wild and resynthesized homoploid and allopolyploid hybrids have revealed widespread changes to gene expression in hybrids which are relative to expression levels in their parents (Veitia, 2005). Many of these changes to gene expression are 'non-additive' and some gene expression changes are far outside the range of gene expression in either parent; therefore, these can be viewed as 'transgressive'. Such profound changes to gene expression may enable new hybrids to survive in novel habitats and not be accessible to their parent species. So explaining the molecular basis of these cases of acquired characters' inheritance has been taxing and an epigenetic perspective may prove to be useful and complementary to conventional genetic explanations. As will be obvious from the key issues of this review (Grant-Downton and Dickinson, 2005), it is 
now evident that the importance of transience versus heritability in epigenetic marks is examined, as these are the potential for stable epigenetic marks that contribute to plant evolution and the mechanisms generating novel epigenetic variation in hybridization and polyploidy.

\section{HYBRID, POLYPLOIDY AND PLANT EVOLUTION}

Hybridization has played a crucial role in the evolution of many plant species, especially to polyploidy. The duplicated genetic material and wide geographical distribution facilitate hybridization and introgression among polyploidy species, having either homologous or analogous genomes. Chances are that, such introgression leads to the production of recombinant genomes which would be more difficult to form at the diploid level. It is reported that, interlocus concerted evolution can occur bidirectional, subsequent to hybridization and polyploidization, which has significant implications for phylogeny reconstruction and the establishment of a geographical subgenus tridentate of Artemisia, biogeography in chelone and a morphological evolution in Brassicaceae (Nelson and Elisens, 1999; Wendel et al., 1995; McArthur and Sanderson, 1999; Lee et al., 2002). Subsequently, a general polyploidy model is found for analyzing gene segregation in and out of the crossing tetraploid species (Wu et al., 2001). However, the details of polyploidy evolution in Dactylorhiza were revealed by amplified fragment length polymorphisms (AFLP) (Hedren et al., 2001), then later, a wild plant genus used a combination of chloroplast and multiple low-copy nuclear loci for phylogenetic inference of polyploidy evolution in Brassicaceae (Slotte et al., 2006), but in some plants, the degree of preferential expression also varies among tissues. Preferential expression was absent in synthetic polyploidy and in some artificial diploid hybrids, it suggested that nucleolar dominance was not necessarily a direct result of hybridization or polyploidization. The establishment of preferential expression in glycine allopolyploids appears to be either stochastic within lineages or genotype specific (Joly et al., 2004). The contributions of domesticated plant studies are used to help our understanding of plant evolution, and it is reported that a wide array of segregating genetic populations will facilitate future research on the evolution and inheritance of quantitative variation in boechera (Hancock, 2005) and spontaneous gene movement from crops to more distantly related species in wheat (Jauhar, 2007; Ishikawa et al., 2009). Nevertheless, between hybridization and polyploidization, which one is best attributed to evolution? It is suggested that, polyploidization rather than hybridization induces genomic changes in tragopogon by cDNA-AFLP polymorphisms. However, hybridization and polyploidyzation have acted as a key evolutionary process in creating a plant group where high-level allopolyploids clearly outnumber extant parental genomes by a network algorithm and non-coding sequences of a low-copy number gene (Brysting et al., 2007). The mechanism of evolution is speculated as a simulation of the evolutionary steps that occurred in nature at the time of the origin of hexaploid wheat (Jauhar, 2007). Recently, molecular evidence of reticulate evolution in the subgenus Plantago by a highly resolved phylogenetic tree (Ishikawa et al., 2009) and general characteristics and classification of haploids derived from diploid and polyploidy species are provided (Jauhar, 2009).

\section{Epigenetic changes conservation in evolution}

Inter-specific hybridization plays an important role in plant adaptive evolution and speciation and the process often results in phenotypic novelty. Hybrids can show changes in genome structure and gene expression when compared with their parents including chromosomal rearrangements and also, changes in cytosine methylation, up- and down-regulation of gene expression and gene silencing emerged. Some of these alterations are not yet explained by Mendelian principles, however, it may have contributed to the evolutionary success of allopolyploids (Finnegan, 2001; Pikaard, 2001; Osborn, 2003) Several hypotheses have been proposed for why gene expression is altered in allopolyploids and hybrids such as DNA methylation (Adams, 2007), which is represented by the conversion of cytosine to 5methylcytosine and causes an important change of DNA in vertebrate and plant genomes (Bird, 1992; GrantDownton and Dickinson, 2005). Recently, significant findings showed that, epigenetic mechanisms may be involved in the stabilization and evolution of nascent allopolyploids (Finnegan, 2001); whereas, one of the more recent lessons about epigenetic responses to hybridization and polyploid formation comes from the genus spartina. MS-AFLP analysis of these hybrids demonstrated that, the genomes of both hybrids have experienced massive methylation and repatterning when compared with their ancestors (Salmon et al., 2005). Moreover, a remarkably high percentage of newly methylated fragments were shared by two hybrids, demonstrating in some sense that the epigenetic reprogramming was 'directed'. Cytosine methylation alterations were induced by inter-specific hybridization between a rice cultivar and its wild relative and indicated a direct relationship between cytosine methylation alteration and gene expression variation using the methylation-sensitive amplified polymorphism (MSAP) method in $\mathrm{F}(1)$ hybrid and $\mathrm{BC}(1)$ progeny following interspecific hybridization between Oryza sativa and Oryza officinalis (Jin et al., 2008). Takamiya et al. (2008) showed that most parental RLGS spots were found in the F1, but eight spots (4\%) showed abnormal inheritance: seven of the eight spots were missing in $\mathrm{F} 1$, and one was 
newly detected in F1. In applicability, the RLGS method was used for the analysis of the inheritance and alteration of methylation in $\mathrm{F} 1$ hybrid plants in the first filial generation (F1) hybrid of $O$. sativa $\mathrm{L}$. ("Nipponbare"x"Kasalath") by restriction landmark genome scanning (RLGS) (Takamiya et al., 2008). Sunao Nakamura and Kazuyoshi Hosaka detected 31 methylation-sensitive RAPD bands in diploid inbred lines of potatoes, of which 11 newly appeared in the self progenies, and 6 of them stably inherited subsequent generations (Nakamura and Hosaka, 2010). Also, differences in cytosine methylation levels demonstrated an epigenetic instability of the allopolyploid of Raphanobrassica between the genetically stable and unstable generations, between Raphanus sativus $\mathrm{L}$. and Brassica alboglabra Bailey (Li, 2010). However, it was found that the changes in cytosine methylation level and pattern were not caused by parental heterozygosity, and they could be either directed or stochastic in a maize hybrid relative to its parental inbred lines (Zhao et al., 2007). Thus, nucleolus size, endopolyploidization level and distribution of DNA and histone $\mathrm{H} 3$ methylation at the microscopic level do not differ between Arabidopsis accessions (Col-0 and C24) and their reciprocal hybrids, and the parental expression profile of the selected genes was maintained in hybrid offspring (Banaei et al., 2010). Aberrant segregations and paternal- or atavism-like transmission were also found in diploid inbred lines of potatoes carlos. The epigenetic nature of the observed flower abnormalities is also consistent with the results and indicates that in the diploid hybrid studies, natural variation in methylation profiles of anonymous DNA sequences could be a biological significance (Marfil et al., 2009) and the combination between inter-specific hybridization and an epigenetically mutagenic treatment like low-dose laser irradiation might be a useful means to generate heritable epigenetic variations in plants (Wang et al., 2010).

Christopher et al. (2008) demonstrated that such epigenetic regulatory variation which exists in recently diverged species suggests a role in reproductive isolation, and this variation is likely to be adaptive (Wiley et al., 2008). Why are some genes silenced, downregulated, or up-regulated in polyploids? Several hypotheses have been proposed with relation to altered regulatory networks and epigenetic remodeling, as well as side effects of other molecular processes, and these hypotheses have been discussed in several articles recently (Wang et al., 2005; Riddle and Birchler, 2003; Adams and Wendel, 2005; Auger et al., 2005; Madlung et al., 2005; Wright et al., 2009). However, the exact mechanism that induces this elimination patterns remains unknown. It seems to be possible that these genomic changes could be as the result of transposon activation in hybrids, resulting in translocations and rearrangements; nonetheless, less dramatic genomic changes of a similar type occur in Arabidopsis suecica by this mechanism (Wang et al., 2005b). The consistency of sequence elimi- nation in these grasses still remains difficult to explain with this mechanism. Other explanations such as more coordinated programmed genomic rearrangements asbeing seen in the programmed DNA excision in ciliates, and a mechanism that depends on the RNA-based pathways (Dolgosheina et al., 2008) should also be taken into consideration. Scascitelli M, Cognet M, Adams KL suggests that novel alternative splicing patterns are presented in a small percentage of genes in hybrids, but they could make a considerable impact on the expression of some genes. Changes in alternative splicing are probably an important component of the genetic changes that occur upon inter-specific hybridization. So it is likely that multiple factors lead to gene expression changes in polyploids and these causes vary by gene and, perhaps, by organism.

\section{Epigenetic changes of plant hybridization}

Classical genetics has revealed the mechanisms for the transmission of genes from generation to generation, but the strategy of the genes in unfolding the developmental programmer remains obscure, such that the non-additive change in genome size and 'transgressive' is caused by hybridization and allopolyploids: epigenetic changes can also play a role (Hegarty et al., 2008).

Molecular studies indicate that epigenetic events are important in determining gene expression. Research in this area has paid particular attention to allopolyploid hybrid, especially in the area of gene silencing. Gene silencing in plants inactivates trans-genes introduced into plants and/or endogenous homologous genes. This stable but potentially reversible loss of gene activity resembles epigenetic changes that occur in normal development.

Epigenetic silencing of a foreign gene was found in nuclear transformants of Chlamydomonas (Cerutti, 1997) and was mediated by Potato virus $X$ amplicons in Arabidopsis (Dalmay et al., 2000).

Petunia plants that exhibit a white-flowering phenotype as a consequence of chalcone synthase transgeneinduced silencing, occasionally give rise to relevant ranches that produce flowers with the wild-type of pigmentation (Kanazawa et al., 2007). Moreover, transposable elements (TEs) are ubiquitously genomic parasites, which implies an evolutionary trade off where the benefit of TE silencing imposes a fitness cost via deleterious effects on the expression of nearby genes (Hollister and Gaut, 2009).

Furthermore, in endogenously homologous genes, mutations in ROR1 reactivate the silenced Pro35S: NPTII gene but not RD29A promoter-luciferase in the ros1 mutant and mutations in the BRU1 gene suggest a novel link between these underlying maintenance mechanisms in Arabidopsis (Takeda et al., 2004; Xia et al., 2006). However, several silenced genes identified in these studies are transcription factors, so gene knock-out tech- 
nology will be an indirect route for gene silencing in allopolyploids.

\section{Epigenetic changes of plant polyploidy}

Polyploidy is remarkably common in the plant kingdom and polyploidization is a major driving force for plant genome evolution. Polyploidy may contain genomes from different parental species (allopolyploids) or include multiple sets of the same genome (autopolyploid). Genetic and epigenetic changes associated with allopolyploidization have been a major research subject in recent years. Polyploidy, or genome doubling, is a common mechanism in the evolution of plants. This genome duplication can rapidly lead to genomic changes between ploidy levels. In particular, allopolyploids resulting from inter-specific hybridization can show a large number of changes in gene expression immediately after the polyploidy event. These are likely due to epigenetic changes that do not alter the underlying DNA sequence. A small number of studies have shown that there may also be some gene expression changes between ploidy levels. They observed the reducing gene expression of the transgenic triploid when compared with diploid hybrids (Scheid et al., 1996). However, it is clearly shown that genome differentiation in allopolyploids is not related to the ploidy level, while the non-additive change in genome size that occurred during allopolyploidization may represent a preprogrammed adaptive response to genomic rigenomes; as such, it is demonstrated that there are few genes, if any, whose expression is linearly correlated with the policy and can be dramatically changed because of policy alteration in potato (Stupar et al., 2007). The patterns and mechanisms of gene regulation during early stages of polyploidy formation were put forward by Wang et al (Wang et al. 2005a). It is indicated that a rapid and stochastic process of differential gene expression is reinforced by epigenetic regulation during polyploidy formation and evolution (Wang et al., 2004). Furthermore, allopolyploidizationaccommodated genomic sequence changes were brought out in triticale (Ma and Gustafson, 2008) and the gene expression was brought out in a wild autopolyploid sunflower series (Church and Spaulding, 2009). A conclusion drawn from the results is that there are not ploidy level-specific differences in gene silencing or novel gene expression. These results support the conclusions of previous studies, that is to say gene expression differences among allopolyploids are likely, in a large part, due to the hybrid nature of these lineages.

\section{MECHANISMS FOR EPIGENETIC CHANGE IN PLANTS}

As we all know, there are three aspects of this problem which would be addressed in plant growth process. The first question involves seed development, while the second problem relates to flower stages and the third aspect deals with fruit maturity. Recent studies of gene expression in polyploid and hybrid plants have examined expression in natural polyploids and synthetic neopolyploids, as well as in diploid and F1 hybrids. Considerable changes in gene expression have been observed in allopolyploids, including up or down regulation of expression in the polyploids compared with their parents, unequal expression of duplicated genes, and silencing of one copy (Adams, 2007; Bateman et al., 2006).

\section{Seed development}

Endosperm tissues account for most of the world's calorific intake, but what regulated the seed development remains to be poorly understood. In 2003, Costa et al. (2003) firstly reported that the globby1-1 (glo1-1) mutation disrupts nuclear and cell division in the developing maize seed causing alterations in endosperm cell fate and tissue differentiation (Costa et al., 2003), while the chromatin assembly factor CAF-1 is required for cellular differentiation during plant development (Exner et al., 2006). Subsequently, a novel gametophytic maternaleffect mutant defective in early embryo and endosperm development, glauce (glc), has been isolated from a population of Arabidopsis Ds transposon insertion lines. It indicates that factors derived from the female activate a subset of the paternal genome of fertilized seeds (Ngo et al., 2007). Recent evidence suggests that signaling processes in both seeds and guard cells involve heterotrimeric $G$ proteins, and these mutants are hypersensitive to abscisic acid regulation of germination and post germination development (Chen et al., 2006). Moreover, the mechanisms for epigenetic change was found by Pien et al. (2008) who reported a novel function of ATX1, namely, the epigenetic regulation of the floral repressor flowering locus C (FLC). They found that ATX1 directly binds the active FLC locus before flowering and this interaction is released upon the transition to flowering (Pien et al., 2008). Nonetheless, such a report as SOC1 negatively regulates the expression of the cold response genes (Seo et al., 2009).

\section{Flower and inflorescence reversion}

Flower and inflorescence reversion was reported to evolve a switch from floral development back to vegetative development, thus rendering flowering a phase in an ongoing growth pattern rather than a terminal act of the meristem. Although it can be considered as an unusual event, the reversion raises questions about the nature and function of flowering. It has a link in environ- 
mental conditions and it is most often a response to conditions that are opposite those that induce flowering. Vernalization is a promotion of the competence for flowering with long periods of low temperatures, such asthose typically experienced during winters. Be it FLC or not, the other side of vernalization is questioned, but it is found that a similar network topology of the apparently independent condition evolved vernalization pathways in grasses and Arabidopsis (Alexandre and Hennig, 2008). In 2005, mechanisms and function of flower and inflorescence reversion was firstly introduced by Tooke et al. (2005) and then the morphological and molecular phylogenetic contexts of the angiosperms contrasted the 'top-down' and 'bottom-up' approaches used to infer the possible characteristics of the first flowers in 2006 (Bateman et al., 2006). Afterwards, Ikeda et al. (2007) found that the molecular basis of late-flowering phenotype was caused by dominant epi-alleles of the FWA locus in Arabidopsis. The previous reports, in which FWA suppressed the precocious-flowering phenotype of plants over expressing flowering locus $T$ (FT), suggest that the flowering pathway(s) either at and/or downstream of FT is blocked by FWA (Ikeda et al., 2007). Moreover, it is suggested that suppressor of FRIGIDA3 encodes a nuclear actin-related protein6 required for floral repression and mutations in the Arabidopsis SWC6 gene, encoding a component of the SWR1 chromatin remodeling complex that accelerates flowering time and alters leaf and flower development (Lazaro et al., 2008; Choi et al., 2005). In addition, small non-coding RNAs are essential to the development of the sporophyte and the somatic diploid phase of flowering plants. A microRNA (miRNA) signal that originated from leaf polarity (Timmermans et al, 2004) and small RNA pathways are presented and functional in the angiosperm male gametophyte (Grant-Downton et al., 2009).

\section{Fruit development}

Variations in early fruit development and composition may have major impacts on the taste and overall quality of the fruit, such as berry color and fleshy fruit by transcription factor. Stommel et al. (2009) found that transcription factor families regulate the anthocyanin biosynthetic pathway in Capsicum annuum (Stommel et al., 2009), while Mounet et al. (2009) advocated it for the first time with the transcriptional and metabolic changes in expanding tomato fruit tissues using multivariate analysis and gene-metabolite correlation networks to get insights into the networks involved in these coordinated processes and to identify key regulatory genes (Mounet et al., 2009). Then, a bud spot which is a somatic mutation in shoot cells of perennial fruits, and a molecular characterization of a bud spot of pinot gris which has bearded white berries were further reported (Furiya et al., 2009). It is said that the maturation and ripening of fleshy fruits is a developmental program that synchronizes seed maturation with metabolism, thereby rendering fruit tissues desirable to seed dispersing organisms. Afterwards, a PLENA-like gene of peach was involved in carpel formation and a subsequent transformation into the fleshy fruit, and then it was found that the fleshy fruit expansion and ripening were regulated by the tomato shatterproof gene TAGL1 (Tadiello et al., 2009; Vrebalov et al, 2009).

\section{Mechanisms for gene expression divergence in hybrid and polyploidy}

Since polyploidy and hybridization are the core of plant evolution, it is critical to identify processes responsible for origins of hybrid species and those that promote shifts in ploidy, changing the possible outcomes of hybrid speciation. Variable patterns of expression according to organ, silencing of genome specific alleles and nonadditive gene expression have all been observed in plant polyploids (Auger et al., 2005; Salmon et al., 2005; Shaked et al., 2001) and were pointed out as consequences of the integration of divergent regulatory hierarchies. There are two important potential ways to perturb gene expression: changes to the genome of a hybrid individual (genetic changes) and epigenetic changes (Hegarty et al. 2008). The heterogeneity of the genomes brought together in plant allopolyploids has been proposed as a major factor underlying patterns of non-additive gene expression, as suggested by studies in different ploidy levels of cotton (Hegarty et al. 2008) and the potential cause-effect relationship between the level of chromosomal (genetic) divergence of the parents and ploidy of hybrids that was recently reviewed by Hegarty et al. (2008) and Buggs et al. (2009). Then, Campbell et al. (2007) demonstrated that, parental nuclear ribosomal internal transcribed spacer (ITS) divergence was significantly greater for allopolyploids than for homoploid hybrids in angiosperms. Buggs and collaborators (Buggs et al., 2009) took a molecular phylogenetic approach to the issue and successfully measured genetic divergence. However, their findings point to a restriction of homoploid formation to parental pairs less divergent than expectation. In these species, the effects of genome merger apparently supplant the impact of genome doubling. Epigenetic changes may reprogram gene expression and developmental patterns of new allopolyploids. Four main types of epigenetic inheritance, namely DNA methylation, chromatin remodelling, genomic imprinting and long-range control by chromatin structure have been described. Changes in DNA methylation frequently occur in the genes of newly formed allopolyploids, as shown by amplified fragment length polymorphism (AFLP) and CDNA-AFLP analyses (Salmon et al., 2005; Comai et al., 2000). It has been widely recognized that cytosine methylation dynamics in 
each generation of animals and the parental methylation states in plants are often stably inherited to sexual progenies (Kakutani et al., 2000). Nevertheless, it was observed in various plant taxa that the formation of interspecific hybrids and allopolyploids is often accompanied by remodeling of the otherwise additive parental methylation patterns (Salmon et al., 2005; Madlung et al., 2002). Parental methylation states of, at least, some genomic loci may also be modified by trans-acting modifiers in certain intra-specific hybrids between different ecotypes in Arabidopsis (Riddle and Richards, 2005) and in several intraspecific maize hybrids (Zhang et al., 2007). All these showed non-Mendelian or epigenetic fashions in inheritance. Therefore, when taken together, this suggests that DNA methylation partly functions epigenetically and dynamically over generations to control and compromise the unbalanced gene expressions caused under certain circumstances (Madlung et al., 2002; Grant-Downton and Dickinson 2006; Zhang et al., 2007; Chan et al., 2005). In addition, chromosomal translocations and transposition (insertion of a DNA fragment into homoeologous chromosomes) are common in Brassica allopolyploids (Song et al., 1995) and changes in gene expression appear to be a major consequence in Arabidopsis and cotton allopolyploids (Adams, 2003; Lee et al., 2006). Moreover, genetic and epigenetic changes may be interrelated (Chen and $\mathrm{Ni}$, 2006); whereas, a reactivation of transposons by chromatin modifications or RNA-mediated pathways may lead to chromosomal breakages and rearrangements. Eliminating DNA sequences (regulatory and/or coding regions) may alter dosage-dependent gene regulation and chromatin structure. Recently, dosage effects on transcription levels was revealed in salmon (Ching et al., 2009) and similar levels of gene expression were reported in triploid and diploid individuals. In the $S$. alburnoides complex, the necessity for balanced gene expression is apparent in both southern (Pala et al., 2008) and northern populations, with higher ploidy forms exhibiting gene expression regulated to the diploid levels, and the expression of one or the other genome was found to depend on the specific genomic combinations that were brought together in the hybrid, irrespective of the level of gene expression (Rapp et al., 2009). In conclusion, the elucidation of the mechanism underlying dosage compensation and preferential allele usage, as well as the characterization of the factors that modulate differential gene expression, will hopefully contribute to a better understanding of how vertebrate polyploid genomes is being regulated and how the different adopted strategies influence their odds on the race for adaptability and evolutionary success.

\section{PROSPECT}

Hybridization between different species and subsequently, polyploidy plays a crucial role in plant genome evolution. Recent molecular genetic studies of hybridization and polyploidy in plants have shown that these critical evolutionary processes have profound effects on patterns of gene expression. Importantly, the changed patterns of gene expression were identified in wild hybrids, indicating that they could contribute to phenotypic phenomena such as hybrid vigor and may be served as a source of variation in hybrids upon which a selection can act. In addition, a greater knowledge of patterns of epigenetic variation will help the study know whether the non-additive and transgressive changes in gene expression in hybrids contribute directly to local adaptation and speciation, and whether it gives it more information in taxonomy and systematic, as well as population biology and conservation. Although important progresses in understanding epigenetic phenomena have been made in the past decade, several open questions remain to be addressed to both better clarify the mechanisms of epigenetics in hybridization and polyploidy, and particularly, to start in applying knowledge from basic research to explore the epigenetic related source of variability deeply. Whether hybridization and polyploidy incite distinct effects with respect to epigenome changes, how different epi-regulatory mechanisms cooperate to regulate global epigenome effects and how each of the epi-regulatory pathways is involved in hybridization and polyploidy, all need to be established further. Moreover, only few epi-regulatory pathways have been characterized at a functional level. So we must generate a collection of tools and information that will constitute the plants platform in hybridization and polyploidy and it is important to establish which epiregulators modulate the transgenerational inheritance of epiallele formation in hybridization and polyploidy: an essential requirement for the generation of epigenetic variability is useful for breeding programs and it is worth mentioning that trans-generational epigenetic modifications, such as paramutations and silent transgenes, are inherited in a relatively high proportion of progeny. Many trans-generational effects by epigenetic modifications, however, may be inherited in a much smaller proportion of the progeny in hybridization and polyploidy. Indeed, the mechanisms of the transgenerational epigenetic modification are largely unknown and need to be elucidated in hybridization and polyploidy. So, the identification of stably inherited epialleles in hybridization and polyploidy, will gain insight into the transgenerational epigenetic modification. It is clear that plants possess novel epigenetic mechanisms compared to animals.

Also, species-specific characteristics exist. However, to what extent species-specific epigenetic marks are related to differences in the genome organization, how they are distinctly contacted in hybridization and polyploidy and how the comparisons of the behavior of hybridization and polyploidy will allow identification of the similarities and 
differences between hybridization and polyploidy are yet to be clarified. In the future, stochastic epimutation and transposon movement will be analyzed through the whole-genome shotgun techniques combined with theBAC-by-BAC approach to cover the entire genome of plants, and the binding sites for epigenetic regulators will be found by development of the ChIP-chip and ChIP-seq technologies. Meanwhile, additional genomic studies will be used to determine when novel genes resulting from polyploidy have enabled adaptive radiations. The deviation in gene expression in hybrids from a purely additive combination of the expression levels of their parents could contribute to phenotypic phenomena such as hybrid vigour, and more importantly, may serve as a source of variation in hybrids upon which selection can act. The most important future goal will determine whether non-additive and transgressive changes in gene expression in hybrids contribute directly to local adaptation and speciation. As such, it should extend and complement the ongoing efforts to research on epigenetic changes in plants. It has been suggested that the recent progress in the area of plant epigenetic changes and plant epigenetic genomics changes have the potential to initiate a series of new revolution. Thus, by integrating the data from cytosine methylation and small RNAs, the chromatin modification in plants would validate the model of comparative epigenomics. The molecular mechanisms for epigenetic change would be demonstrated and would thus elucidate the control seed development, abioticinduced and biotic-induced signaling pathway, respectively. Therefore, it will help to cultivate new plants breeds, such as drought tolerance breeds, pest-resistant breeds and transgene breeds.

\section{ACKNOWLEDGEMENTS}

This work was supported by the Program for Science and Technology Department Application Foundation of Sichuan Province (2006J13-039), Changjiang Scholars and Innovative Research Team in the University of China (Grant No. IRT-0453), the Program for the Ministry of Agriculture of the People's Republic of China (2003-Q03), the Program for the Ministry of Science and Technology of the People's Republic of China (2006BAD13B03) and the High-tech Research and Development Program of China (2006AA100103).

\section{REFERENCES}

Adams KL, Wendel JF (2005). Polyploidy and genome evolution in plants. Current Opinion in Plant Biology, 8(2):135-141.

Adams KL (2007). Evolution of Duplicate Gene Expression in Polyploid and Hybrid Plants. J Hered. 98(2):136-141.

Adams KL (2003). Genes duplicated by polyploidy show unequal contributions to the transcriptome and organ-specific reciprocal silencing. Proceedings of the National Academy of Sciences, 100(8):4649-4654.

Alexandre CM, Hennig L (2008). FLC or not FLC: the other side of vernalization. J Exp Bot. 59(6):1127-1135.

Auger DL, Gray AD, Ream TS, Kato A, Coe EH, Jr., Birchler JA (2005). Nonadditive Gene Expression in Diploid and Triploid Hybrids of Maize. Genetics, 169(1):389-397.

Auger DL, Peters EM, Birchler JA(2005). A genetic test of bioactive gibberellins as regulators of heterosis in maize. J. Hered. 96(5):614-617.

Banaei Moghaddam A, Fuchs J, Czauderna T, Houben A, Mette M (2010). Intraspecific hybrids of \&lt;i\&gt;Arabidopsis thaliana\&lt;/i\&gt; revealed no gross alterations in endopolyploidy, DNA methylation, histone modifications and transcript levels. TAG Theoretical and Applied Genetics, 120(2):215-226.

Bateman RM, Hilton J, Rudall PJ (2006). Morphological and molecular phylogenetic context of the angiosperms: contrasting the 'top-down' and 'bottom-up' approaches used to infer the likely characteristics of the first flowers. J Exp Bot. 57(13):3471-3503.

Bird A (1992). The essentials of DNA methylation. Cell, 70(1):5-8.

Brysting AK, Oxelman B, Huber KT, Moulton V, Brochmann C (2007). Untangling Complex Histories of Genome Mergings in High Polyploids. Syst. Biol. 56(3):467-476.

Buggs RJA, Doust AN, Tate JA, Koh J, Soltis K, Feltus FA, Paterson AH, Soltis PS, Soltis DE (2009). Gene loss and silencing in Tragopogon miscellus (Asteraceae): comparison of natural and synthetic allotetraploids. Heredity, 103(1):73-81.

Campbell DR, Chapman KE, Waldron KJ, Tottey S, Kendall S, Cavallaro G, Andreini C, Hinds J, Stoker NG, Robinson NJ, Cavet JS (2007). Mycobacterial Cells Have Dual Nickel-Cobalt Sensors: Sequence Relationships And Metal Sites Of Metal-Responsive Repressors Are Not Congruent. J. Biol. Chem. 282(44):32298-32310.

Cerutti H, Johnson AM, Gillham NW, Boynton JE (1997). Epigenetic Silencing of a Foreign Gene in Nuclear Transformants of Chlamydomonas. Plant Cell, 9(6):925-945.

Chan GC, Fish JE, Mawji IA, Leung DD, Rachlis AC, Marsden PA (2005). Epigenetic Basis for the Transcriptional Hyporesponsiveness of the Human Inducible Nitric Oxide Synthase Gene in Vascular Endothelial Cells. J. Immunol, 175(6):3846-3861.

Chen ZJ, Ni Z (2006). Mechanisms of genomic rearrangements and gene expression changes in plant polyploids. Bioessays, 28(3):240252.

Ching B, Jamieson S, Heath JW, Heath DD, Hubberstey A (2009). Transcriptional differences between triploid and diploid Chinook salmon (Oncorhynchus tshawytscha) during live Vibrio anguillarum challenge. Heredity, 104(2):224-234.

Choi K, Kim S, Kim SY, Kim M, Hyun Y, Lee H, Choe S, Kim S-G, Michaels S, Lee I (2005). Suppressor of frigida3 encodes a nuclear actin-related protein 6 required for floral repression in arabidopsis. Plant Cell, 17(10):2647-2660.

Church SA, Spaulding EJ (2009). Gene Expression in a Wild Autopolyploid Sunflower Series. J. Hered. 100(4):491-495.

Comai L, Tyagi AP, Winter K, Holmes-Davis R, Reynolds SH, Stevens Y, Byers B (2000). Phenotypic Instability and Rapid Gene Silencing in Newly Formed Arabidopsis Allotetraploids. Plant Cell, 12(9):15511568.

Costa LM, Gutierrez-Marcos JF, Brutnell TP, Greenland AJ, Dickinson HG (2003). The globby1-1 (glo1-1) mutation disrupts nuclear and cell division in the developing maize seed causing alterations in endosperm cell fate and tissue differentiation. Development, 130(20):5009-5017.

Dalmay T, Hamilton A, Mueller E, Baulcombe DC (2000). Potato Virus X Amplicons in Arabidopsis Mediate Genetic and Epigenetic Gene Silencing. Plant Cell, 12(3):369-380.

Dolgosheina EV, Morin RD, Aksay G, Sahinalp SC, Magrini V, Mardis ER, Mattsson J, Unrau PJ (2008). Conifers have a unique small RNA silencing signature. RNA, 14(8):1508-1515.

Exner V, Taranto P, Schonrock N, Gruissem W, Hennig L (2006). Chromatin assembly factor CAF-1 is required for cellular differentiation during plant development. Development, 133(21):41634172.

Finnegan EJ (2001). Is plant gene expression regulated globally? Trends in genetics: TIG, 17(7):361-365.

Furiya T, Suzuki S, Sueta T, Takayanagi T (2009). Molecular Characterization of a Bud Sport of Pinot gris Bearing White Berries. 
Am. J. Enol. Vitic, 60(1):66-73.

Grant-Downton R, Hafidh S, Twell D, Dickinson HG (2009). Small RNA Pathways Are Present and Functional in the Angiosperm Male Gametophyte. Mol Plant, 2(3):500-512.

GRANT-DOWNTON RT, DICKINSON HG (2006). Epigenetics and its implications for Plant Biology 2. The 'Epigenetic Epiphany': Epigenetics, Evolution and Beyond. Ann. Bot. 97(1):11-27.

GRANT-DOWNTON RT, DICKINSON HG (2005). Epigenetics and its Implications for Plant Biology. 1. The Epigenetic Network in Plants. Ann Bot, 96(7):1143-1164.

HANCOCK JF (2005). Contributions of Domesticated Plant Studies to our Understanding of Plant Evolution. Ann. Bot. 96(6):953-963.

Hedren M, Fay MF, Chase MW (2001). Amplified fragment length polymorphisms (AFLP) reveal details of polyploid evolution in Dactylorhiza (Orchidaceae). Am J Bot. 88(10):1868-1880.

Hegarty MJ, Barker GL, Brennan AC, Edwards KJ, Abbott RJ, Hiscock SJ (2008). Changes to gene expression associated with hybrid speciation in plants: further insights from transcriptomic studies in Senecio. Philo. Trans. R. Soc. Lond. B. Bio. Sci. 363(1506):30553069.

Hollister JD, Gaut BS (2009). Epigenetic silencing of transposable elements: A trade-off between reduced transposition and deleterious effects on neighboring gene expression. Genome Res. 19(8):14191428.

Ikeda Y, Kobayashi Y, Yamaguchi A, Abe M, Araki T (2007). Molecular basis of late-flowering phenotype caused by dominant epi-alleles of the FWA locus in Arabidopsis. Plant Cell Physiol. 48(2):205-220.

Ishikawa N, Yokoyama J, Tsukaya H (2009). Molecular evidence of reticulate evolution in the subgenus Plantago (Plantaginaceae). Am. J. Bot. 96(9):1627-1635.

Jauhar PP, Xu SS, Baenziger PS (2009). Haploidy in Cultivated Wheats: Induction and Utility in Basic and Applied Research. Crop Sci. 49(3):737-755.

Jauhar PP (2007). Meiotic Restitution in Wheat Polyhaploids (Amphihaploids): A Potent Evolutionary Force. J. Hered, 98(2):188193.

Jin H, Hu W, Wei Z, Wan L, Li G, Tan G, Zhu L, He G (2008). Alterations in cytosine methylation and species-specific transcription induced by interspecific hybridization between \&lt;i\&gt;Oryza sativa\&lt;/i\&gt; and \&lt;i\&gt;O. officinalis\&lt;/i\&gt. TAG Theoretical and Applied Genetics, 117(8):1271-1279.

Joly S, Rauscher JT, Sherman-Broyles SL, Brown AHD, Doyle JJ (2004). Evolutionary Dynamics and Preferential Expression of Homeologous 18S-5.8S-26S Nuclear Ribosomal Genes in Natural and Artificial Glycine Allopolyploids. Mol. Bio. Evol. 21(7):1409-1421.

Kakutani T, Munakata K, Richards EJ, Hirochika H (2000). SUV39H1 Drives Chromatin Structure Meiotically and Mitotically Stable Inheritance of DNA Hypomethylation Induced by ddm1 Mutation of Arabidopsis thaliana. Sci STKE, 2000(45):tw2-.

Kanazawa A, O'Dell M, Hellens RP (2007). Epigenetic Inactivation of Chalcone Synthase-A Transgene Transcription in Petunia Leads to a Reversion of the Post-Transcriptional Gene Silencing Phenotype. Plant Cell Physiol. 48(4):638-647.

Lazaro A, Gomez-Zambrano A, Lopez-Gonzalez L, Pineiro M, Jarillo JA (2008). Mutations in the Arabidopsis SWC6 gene, encoding a component of the SWR1 chromatin remodelling complex, accelerate flowering time and alter leaf and flower development. J. Exp. Bot. 59(3):653-666.

Lee H-R, Neumann P, Macas J, Jiang J (2006). Transcription and Evolutionary Dynamics of the Centromeric Satellite Repeat CentO in Rice. Mol. Bio. Evol. 23(12):2505-2520.

Lee J-Y, Mummenhoff K, Bowman JL (2002). Allopolyploidization and evolution of species with reduced floral structures in Lepidium L. (Brassicaceae). PNAS, 99(26):16835-16840.

Li X, Guo W, Wang B, Li X, Chen H, Wei L, Wang Y, Wu J, Long $\mathrm{H}$ (2010). Instability of chromosome number and DNA methylation variation induced by hybridization and amphidiploid formation between Raphanus sativus L. and Brassica alboglabra Bailey. BMC Plant Bio. 10(1):207.

Ma X-F, Gustafson JP (2008). Allopolyploidization-accommodated Genomic Sequence Changes in Triticale. Ann. Bot. 101(6):825-832.

Madlung A, Masuelli RW, Watson B, Reynolds SH, Davison J, Comai L
(2002). Remodeling of DNA Methylation and Phenotypic and Transcriptional Changes in Synthetic Arabidopsis Allotetraploids. Plant Physiol. 129(2):733-746.

Madlung A, Tyagi AP, Watson B, Jiang $H$, Kagochi T, Doerge RW, Martienssen R, Comai $L$ (2005).Genomic changes in synthetic Arabidopsis polyploids. Plant J. 41(2):221-230.

Marfil CF, Camadro EL, Masuelli RW (2009). Phenotypic instability and epigenetic variability in a diploid potato of hybrid origin, Solanum ruizlealii. BMC Plant Bio., 9:21.

McArthur ED, Sanderson SC (1999). Cytogeography and chromosome evolution of subgenus Tridentatae of Artemisia (Asteraceae). Am. J. Bot. 86(12):1754-1775.

Mounet F, Moing A, Garcia V, Petit J, Maucourt M, Deborde C, Bernillon S, Le Gall G, Colquhoun I, Defernez M, Giraudel J-L, Rolin D, Rothan C, Lemaire-Chamley M (2009). Gene and Metabolite Regulatory Network Analysis of Early Developing Fruit Tissues Highlights New Candidate Genes for the Control of Tomato Fruit Composition and Development. Plant Physiol. 149(3):1505-1528.

Nakamura S, Hosaka K (2010). DNA methylation in diploid inbred lines of potatoes and its possible role in the regulation of heterosis. Theor. Appl. Genet. 120(2):205-214.

Nelson AD, Elisens WJ (1999). Polyploid evolution and biogeography in Chelone (Scrophulariaceae): morphological and isozyme evidence. Am. J. Bot. 86(10):1487-1501.

Ngo QA, Moore JM, Baskar R, Grossniklaus U, Sundaresan V (2007). Arabidopsis GLAUCE promotes fertilization-independent endosperm development and expression of paternally inherited alleles. Development, 134(22):4107-4117.

Osborn T (2003). Understanding mechanisms of novel gene expression in polyploids. Trends in Genetics, 19(3):141-147.

Ozkan H, Tuna M, Arumuganathan K (2003). Nonadditive Changes in Genome Size During Allopolyploidization in the Wheat (AegilopsTriticum) Group. J. Hered. 94(3):260-264.

Pala I, Coelho MM, Schartl M (2008). Dosage compensation by genecopy silencing in a triploid hybrid fish. Curr. Bio. 18(17):1344-1348.

Pien S, Fleury D, Mylne JS, Crevillen P, Inze D, Avramova Z, Dean C, Grossniklaus U (2008). Arabidopsis Trithorax1 Dynamically Regulates flowering locus C Activation via Histone 3 Lysine 4 Trimethylation. Plant Cell, 20(3):580-588.

Pikaard CS (2001). Genomic change and gene silencing in polyploids. Trends Genet, 17(12):675-677.

Rapp RA, Udall JA, Wendel JF (2009). Genomic expression dominance in allopolyploids. BMC Biol. 7:18.

Riddle NC, Birchler JA (2003). Effects of reunited diverged regulatory hierarchies in allopolyploids and species hybrids. Trends in Genetics, 19(11):597-600.

Riddle NC, Richards EJ (2005). Genetic variation in epigenetic inheritance of ribosomal RNA gene methylation in Arabidopsis. Plant J. 41(4):524-532.

Salmon A, Ainouche ML, Wendel JF (2005). Genetic and epigenetic consequences of recent hybridization and polyploidy in Spartina (Poaceae). Molecular Ecol. 14(4):1163-1175.

Scheid OM, Jakovleva L, Afsar K, Maluszynska J, Paszkowski J (1996). A change of ploidy can modify epigenetic silencing. PNAS, 93(14):7114-7119.

Seo E, Lee H, Jeon J, Park H, Kim J, Noh Y-S, Lee I (2009). Crosstalk between Cold Response and Flowering in Arabidopsis Is Mediated through the Flowering-Time Gene SOC1 and Its Upstream Negative Regulator FLC. Plant Cell, 21(10):3185-3197.

Shaked H, Kashkush K, Ozkan H, Feldman M, Levy AA (2001). Sequence Elimination and Cytosine Methylation Are Rapid and Reproducible Responses of the Genome to Wide Hybridization and Allopolyploidy in Wheat. Plant Cell, 13(8):1749-1759.

Slotte T, Ceplitis A, Neuffer B, Hurka H, Lascoux M (2006). Intrageneric phylogeny of Capsella (Brassicaceae) and the origin of the tetraploid C. bursa-pastoris based on chloroplast and nuclear DNA sequences. Am. J. Bot. 3(11):1714-1724.

Song K, Lu P, Tang K, Osborn TC (1995). Rapid genome change in synthetic polyploids of Brassica and its implications for polyploid evolution. Proc. Nat. Acad. Sci. USA, 92(17):7719-7723.

Stommel JR, Lightbourn GJ, Winkel BS, Griesbach RJ (2009). Transcription Factor Families Regulate the Anthocyanin Biosynthetic 
Pathway in Capsicum annuum. J. Am. Soc. Hort. Sci. 134(2):244251.

Stupar RM, Bhaskar PB, Yandell BS, Rensink WA, Hart AL, Ouyang S, Veilleux RE, Busse JS, Erhardt RJ, Buell CR, Jiang J (2007). Phenotypic and Transcriptomic Changes Associated With Potato Autopolyploidization. Genetics, 176(4): 2055-2067.

Tadiello A, Pavanello A, Zanin D, Caporali E, Colombo L, Rotino GL, Trainotti L, Casadoro G (2009). A PLENA-like gene of peach is involved in carpel formation and subsequent transformation into a fleshy fruit. J. Exp. Bot. 60(2):651-661.

Takamiya T, Hosobuchi S, Noguchi T, Asai K, Nakamura E, Habu Y, Paterson AH, lijima H, Murakami Y, Okuizumi H (2008). Inheritance and alteration of genome methylation in $\mathrm{F} 1$ hybrid rice. Electrophoresis, 29(19):4088-4095.

Takeda S, Tadele Z, Hofmann I, Probst AV, Angelis KJ, Kaya H, Araki T, Mengiste T, Scheid OM, Shibahara K-i et al (2004). BRU1, a novel link between responses to DNA damage and epigenetic gene silencing in Arabidopsis. Genes \&amp; Dev. 18(7):782-793.

TIMMERMANS MCP, JUAREZ MT, PHELPS-DURR TL (2004). A Conserved microRNA Signal Specifies Leaf Polarity. Cold Spring Harb. Symp. Quant. Biol. 69(0):409-418.

Tooke F, Ordidge M, Chiurugwi T, Battey N (2005). Mechanisms and function of flower and inflorescence reversion. J. Exp. Bot. 56(420):2587-2599.

Veitia RA (2005). Paralogs in Polyploids: One for All and All for One? Plant Cell, 17(1):4-11.

Vrebalov J, Pan IL, Arroyo AJ, McQuinn R, Chung M, Poole M, Rose J, Seymour G, Grandillo S, Giovannoni J, Irish VF (2009). Fleshy Fruit Expansion and Ripening Are Regulated by the Tomato Shatterproof Gene TAGL1. Plant Cell, 21(10):3041-3062.

Wang H, Feng Q, Zhang M, Yang C, Sha W, Liu B (2010). Alteration of DNA methylation level and pattern in sorghum (Sorghum bicolor L.) pure-lines and inter-line F1 hybrids following low-dose laser irradiation. J. Photochem. Photobio. B, 99(3):150-153.

Wang J, Tian L, Lee HS, Wei N, Jiang H, Watson B, Madlung A, Osborn $\mathrm{T}$, Doerge R, Comai L (2005a). Genome-wide non-additive gene regulation in Arabidopsis allotetraploids. Genetics, 105.047894.

Wang J, Tian L, Lee H-S, Wei N, Jiang H, Watson B, Madlung A, Osborn T, Doerge RD, Comai L, Chen ZJ (2005b). Genome-wide Non-additive Gene Regulation in Arabidopsis allotetraploids. Genetics, 105.047894.

Wang J, Tian L, Madlung A, Lee H-S, Chen M, Lee JJ, Watson B, Kagochi T, Comai L, Chen ZJ (2004). Stochastic and Epigenetic Changes of Gene Expression in Arabidopsis Polyploids. Genetics, 167(4):1961-1973.
Wendel J, Schnabel A, Seelanan T (1995).Bidirectional Interl ocus Concerted Evolution Following Allopolyploid Speciation in Cotton (Gossypium). PNAS, 92(1):280-284.

Wiley CD, Matundan HH, Duselis AR, Isaacs AT, Vrana PB (2008). Patterns of hybrid loss of imprinting reveal tissue- and cluster-specific regulation. PLoS One, 3(10):e3572.

Wright KM, Pires JC, Madlung A (2009). Mitotic instability in resynthesized and natural polyploids of the genus Arabidopsis (Brassicaceae). Am. J. Bot. 96(9):1656-1664.

Wu R, Gallo-Meagher M, Littell RC, Zeng Z-B (2001). A General Polyploid Model for Analyzing Gene Segregation in Outcrossing Tetraploid Species. Genetics, 159(2):869-882.

Xia R, Wang J, Liu C, Wang Y, Wang Y, Zhai J, Liu J, Hong X, Cao X, Zhu J-K (2006). ROR1/RPA2A, a Putative Replication Protein A2, Functions in Epigenetic Gene Silencing and in Regulation of Meristem Development in Arabidopsis. Plant Cell, 18(1):85-103.

Zhang MS, Yan HY, Zhao N, Lin XY, Pang JS, Xu KZ, Liu LX, Liu B (2007). Endosperm-specific hypomethylation, and meiotic inheritance and variation of DNA methylation level and pattern in sorghum (Sorghum bicolor L.) inter-strain hybrids. Theor. Appl. Genet. 115(2):195-207.

Zhao X, Chai Y, Liu B (2007). Epigenetic inheritance and variation of DNA methylation level and pattern in maize intra-specific hybrids. Plant Sci. 172(5):930-938. 